This postprint is published in:

Topological Methods in Nonlinear Analysis, Volumen 51, Number 1, March 2018, 55-77

DOI: 10.12775/TMNA.2017.037

\title{
NONHOMOGENEOUS DIRICHLET PROBLEMS WITHOUT THE AMBROSETTI-RABINOWITZ CONDITION
}

\author{
GANG LI, VICENŢIU D. RĂDULESCU, DUŠAN D. REPOVŠ AND QIHU ZHANG
}

\begin{abstract}
We consider the existence of solutions of the following $p(x)$-Laplacian Dirichlet problem without the Ambrosetti-Rabinowitz condition:

$$
\left\{\begin{array}{l}
-\operatorname{div}\left(|\nabla u|^{p(x)-2} \nabla u\right)=f(x, u), \text { in } \Omega \\
u=0, \text { on } \partial \Omega
\end{array}\right.
$$

We give a new growth condition and we point out its importance for checking the Cerami compactness condition. We prove the existence of solutions of the above problem via the critical point theory, and also provide some multiplicity properties. Our results extend previous results of Q. Zhang and C. Zhao (Existence of strong solutions of a $p(x)$-Laplacian Dirichlet problem without the Ambrosetti-Rabinowitz condition, Computers and Mathematics with Applications, 2015) and we establish the existence of solutions under weaker hypotheses on the nonlinear term.
\end{abstract}

\section{INTRODUCTION}

In recent years, the study of differential equations and variational problems with variable exponent growth conditions has been a topic of great interest. This type of problems has very strong background, for instance in image processing, nonlinear electro-rheological fluids and elastic mechanics. Some of these phenomena are related to the Winslow effect, which describes the behavior of certain fluids that become solids or quasi-solids when subjected to an electric field. The result was named after the American engineer Willis M. Winslow.

There are many papers dealing with problems with variable exponents, see [1]-[8], [10]-[25], [28], [33]-[34], [37], [38], [40]-[46], [48]-[49]. On the existence of solutions of these kinds of problems, we refer to $[8,14,15,18,21,33,36,45]$. We also refer to the recent monograph [35] dealing with variational methods in the framework of nonlinear problems with variable exponent.

In this paper, we consider the existence of solutions of the following class of Dirichlet problems:

$$
\text { (P) }\left\{\begin{array}{l}
-\Delta_{p(x)} u:=-\operatorname{div}\left(|\nabla u|^{p(x)-2} \nabla u\right)=f(x, u), \quad \text { in } \Omega, \\
u=0, \quad \text { on } \partial \Omega,
\end{array}\right.
$$

where $\Omega \subset \mathbb{R}^{N}$ is a bounded domain with $C^{1, \alpha}$ smooth boundary, and $p(\cdot)>1$ is of class $C^{1}(\bar{\Omega})$.

Key words and phrases. $p(x)$-Laplace operator, Dirichlet problem, Ambrosetti-Rabinowitz condition, variable exponent space, critical point.

2010 Mathematics Subject Classification. Primary: 35J60. Secondary: 35J20, 35J25, 58E05. 
Since the elliptic operator with variable exponent is not homogeneous, new methods and techniques are needed to study these types of problems. We point out that commonly known methods and techniques for studying constant exponent equations fail in the setting of problems involving variable exponents. For instance, the eigenvalues of the $p(x)$-Laplacian Dirichlet problem were studied in [16]. In this case, if $\Omega \subset \mathbb{R}^{N}$ is a smooth bounded domain, then the Rayleigh quotient

$$
\lambda_{p(\cdot)}=\inf _{u \in W_{0}^{1, p(\cdot)}(\Omega) \backslash\{0\}} \frac{\int_{\Omega} \frac{1}{p(x)}|\nabla u|^{p(x)} d x}{\int_{\Omega} \frac{1}{p(x)}|u|^{p(x)} d x}
$$

is in general zero, and $\lambda_{p(\cdot)}>0$ holds only under some special conditions.

In [41], the author generalized the Picone identities for half-linear elliptic operators with $p(x)$-Laplacian. In the same paper some applications to Sturmian comparison theory are also presented, but the formula is different from the constant exponent case. In a related setting, we point out that the formula

$$
\int_{\Omega}|u(x)|^{p} d x=p \int_{0}^{\infty} t^{p-1}|\{x \in \Omega ;|u(x)|>t\}| d t
$$

has no variable exponent analogue.

In [23] and [46] the authors deal with the local boundedness and the Harnack inequality for the $p(x)$-Laplace equation. But it was shown in [23] that even in the case of a very nice exponent, for example,

$$
p(x):= \begin{cases}3, & \text { for } 0<x \leq \frac{1}{2} \\ 3-2\left(x-\frac{1}{2}\right), & \text { for } \frac{1}{2}<x<1\end{cases}
$$

the constant in the Harnack inequality depends on the minimizer, that is, the inequality $\sup u \leq c \inf u$ does not hold for any absolute constant $c$.

The standard norm in variable exponent Sobolev spaces is the so-called Luxemburg norm $|u|_{p(\cdot)}$ (see section 2) and the integral $\int_{\Omega}|u(x)|^{p(x)} d x$ does not satisfy the constant power relation.

In many instances, it is difficult to judge whether or not results about $p$-Laplacian can be generalized to $p(x)$-Laplacian, and even if this can be done, it is still difficult to figure out the form in which the results should be.

Our main goal is to obtain a couple of existence results for the problem $(\mathrm{P})$ without the Ambrosetti-Rabinowitz condition via critical point theory. For this purpose, we use a new method for checking the Cerami compactness condition under a new growth condition. Our results can be regarded as extensions of the corresponding results for the $p$-Laplacian problems, but the growth condition and the methods for checking the Cerami compactness condition are different with respect to quasilinear equations with constant exponent. 
Next, we give a review of some results related to our work. Since the AmbrosettiRabinowitz type condition is quite restrictive and excludes many cases of nonlinearity, there are many papers dealing with the problem without the AmbrosettiRabinowitz type growth condition. For the constant exponent case $p(\cdot) \equiv p$, we refer to $[26,27,31,39]$.

In [26], the authors considered the problem $(\mathrm{P})$ for $p(\cdot) \equiv p$, and proved the existence of weak solutions under the following assumptions: $\lim _{|t| \rightarrow+\infty} \frac{F(x, t)}{|t|^{p}}=+\infty$, where $F(x, t)=\int_{0}^{t} f(x, s) d s$; and there exists a constant $C_{*}>0$ such that $H(x, t) \leq$ $H(x, s)+C_{*}$ for each $x \in \Omega, 0<t<s$ or $s<t<0$, where $H(x, t)=t f(x, t)-$ $p F(x, t)$.

In [27], the author studied the problem $(\mathrm{P})$ for $p(\cdot) \equiv p$. Under the assumption that $\frac{f(x, s)}{|s|^{p-2} s}$ is increasing when $s \geq s_{0}$ and decreasing when $s \leq-s_{0}, \forall x \in \Omega$, the existence of weak solutions was obtained.

In [31], the authors studied the problem $(\mathrm{P})$ for $p(\cdot) \equiv 2$, which becomes a Laplacian problem. The main result in [31] establishes the existence of weak solutions by assuming that $\frac{f(x, s)}{s}$ is increasing when $s \geq s_{0}$ and decreasing when $s \leq-s_{0}$, for all $x \in \Omega$.

In [39], the author also studied the problem $(\mathrm{P})$ for $p(\cdot) \equiv 2$ and proved the existence of weak solutions under the assumption

$$
s f(x, u) \geq C_{0}|s|^{\mu} \text {, where } \mu>2 \text { and } C_{0}>0 .
$$

If $p(\cdot)$ is a general function, results on variable exponent problem without the Ambrosetti-Rabinowitz type growth condition are rare due to the complexity of $p(x)$-Laplacian (see $[3,5,19,20,42])$. However their assumptions imply $G_{p^{+}}(x, t)=$ $f(x, t) t-p^{+} F(x, t) \geq 0$ and $F(x, t)>0$ as $t \rightarrow+\infty$, so we can see that $F(x, t) \geq$ $C t^{p^{+}}$as $t \rightarrow+\infty$. This is too strong and unnatural for the $p(x)$-Laplacian problems.

In [45], the author considered the problem $(\mathrm{P})$ under the following growth condition:

there exist constants $M, C_{1}, C_{2}>0, a>p$ on $\bar{\Omega}$ such that

$C_{1}|t|^{p(x)}[\ln (e+|t|)]^{a(x)-1} \leq C_{2} \frac{t f(x, t)}{\ln (e+|t|)} \leq t f(x, t)-p(x) F(x, t), \forall|t| \geq M, \forall x \in \Omega$.

A typical example is $f(x, t)=|t|^{p(x)-2} t[\ln (1+|t|)]^{a(x)}$. This function satisfies the above condition (2), but does not satisfy the Ambrosetti-Rabinowitz condition.

Our paper was motivated by [45]. We further weaken the condition (2). To begin we point out that the assumption $a>p$ on $\bar{\Omega}$ is unnecessary in the present paper.

Before stating our main results, we make the following assumptions:

$\left(\mathrm{f}_{0}\right): f: \Omega \times \mathbb{R} \rightarrow \mathbb{R}$ satisfies the Carathéodory condition and

$$
|f(x, t)| \leq C\left(1+|t|^{\alpha(x)-1}\right), \forall(x, t) \in \Omega \times \mathbb{R},
$$

where $\alpha \in C(\bar{\Omega})$ and $p(x)<\alpha(x)<p^{*}(x)$ on $\bar{\Omega}$. 
$\left(\mathrm{f}_{1}\right)$ : there exist constants $M, C>0$, such that

$$
C \frac{t f(x, t)}{K(t)} \leq t f(x, t)-p(x) F(x, t), \forall|t| \geq M, \forall x \in \bar{\Omega},
$$

and

$$
\frac{t f(x, t)}{|t|^{p(x)}[K(t)]^{p(x)}} \rightarrow+\infty \text { uniformly as }|t| \rightarrow+\infty \text { for } x \in \bar{\Omega},
$$

where $K$ satisfies the following hypotheses:

$(\mathrm{K}): 1 \leq K(\cdot) \in C^{1}([0,+\infty),[1,+\infty))$ is increasing and $[\ln (e+t)]^{2} \geq K(t) \rightarrow+\infty$ as $|t| \rightarrow+\infty$, which satisfies $t K^{\prime}(t) / K(t) \leq \sigma_{0} \in(0,1)$, where $\sigma_{0}$ is a constant.

$\left(\mathrm{f}_{2}\right): f(x, t)=o\left(|t|^{p(x)-1}\right)$ uniformly for $x \in \Omega$ as $t \rightarrow 0$.

$\left(\mathrm{f}_{3}\right): f(x,-t)=-f(x, t), \forall x \in \bar{\Omega}, \forall t \in \mathbb{R}$.

$\left(\mathrm{f}_{4}\right): F$ satisfies

$$
\frac{F(x, t)}{|t|^{p(x)}[\ln (e+|t|)]^{p(x)}} \rightarrow+\infty \text { uniformly as }|t| \rightarrow+\infty \text { for } x \in \bar{\Omega} .
$$

$\left(\mathrm{p}_{1}\right)$ : there is a vector $l \in \mathbb{R}^{N} \backslash\{0\}$ such that for any $x \in \Omega, \rho(t)=p(x+t l)$ is monotone for $t \in I_{x}(l)=\{t \mid x+t l \in \Omega\}$.

$\left(\mathrm{p}_{2}\right): p$ has a local maximum point, that is, there exist $x_{0} \in \Omega$ and $\delta>0$ such that $\overline{B\left(x_{0}, 3 \delta\right)} \subset \Omega$ and

$$
\min _{\left|x-x_{0}\right| \leq \delta} p(x)>\max _{2 \delta \leq\left|x-x_{0}\right| \leq 3 \delta} p(x) .
$$

$\left(\mathrm{p}_{3}\right): p$ has a sequence of local maximum points, that is, there exist a sequence of points $x_{n} \in \Omega$ and $\delta_{n}>0$ such that $\overline{B\left(x_{0}, 3 \delta_{n}\right)}$ are mutually disjoint and

$$
\min _{\left|x-x_{n}\right| \leq \delta_{n}} p(x)>\max _{2 \delta_{n} \leq\left|x-x_{n}\right| \leq 3 \delta_{n}} p(x) .
$$

We state our main results in what follows.

Theorem 1.1 Assume that hypotheses $\left(f_{0}\right)-\left(f_{2}\right),\left(p_{1}\right)$, and $\left(f_{4}\right)$ or $\left(p_{2}\right)$ are fulfilled. Then problem $(\mathrm{P})$ has a nontrivial solution.

Theorem 1.2 Assume that hypotheses $\left(f_{0}\right),\left(f_{1}\right),\left(f_{3}\right)$, and $\left(f_{4}\right)$ or $\left(p_{3}\right)$ are fulfilled. Then problem $(\mathrm{P})$ has infinitely many pairs of solutions.

Remark. (i). The following functions satisfy the hypothesis $(K)$ :

$$
\begin{aligned}
& K_{1}(t)=\ln (e+|t|) \\
& K_{2}(t)=\ln (e+\ln (e+|t|)) \\
& K_{3}(t)=[\ln (e+\ln (e+|t|))] \ln (e+|t|) .
\end{aligned}
$$

Let $K=K_{1}$, and $f(x, t)=|t|^{p(x)-2} t[\ln (1+|t|)]^{p(x)} \rho(|t|)$, where $1 \leq \rho(|t|) \leq$ $[\ln (e+|t|)]^{2}, \rho^{\prime} \geq 0$ and $\rho(|t|) \rightarrow+\infty$ as $|t| \rightarrow+\infty$, for example $\rho(|t|)=\ln (e+$ $\ln (e+|t|))$. Then $f$ satisfies the condition $\left(\mathrm{f}_{0}\right)-\left(\mathrm{f}_{4}\right)$, but it does not satisfy the Ambrosetti-Rabinowitz condition, and does not satisfy (2) (ii). We do not need any monotonicity assumption on $f(x, \cdot)$. 
This paper is organized as follows. In Section 2, we do some preparatory work including some basic properties of the variable exponent Sobolev spaces, which can be regarded as a special class of generalized Orlicz-Sobolev spaces. In Section 3, we give proofs of the results stated above.

\section{Preliminary Results}

Throughout this paper, we use letters $c, c_{i}, C, C_{i}, i=1,2, \ldots$ to denote generic positive constants which may vary from line to line, and we will specify them whenever necessary.

One of the reasons for the huge development of the theory of classical Lebesgue and Sobolev spaces $L^{p}$ and $W^{1, p}$ (where $1 \leq p \leq \infty$ ) is its usefulness for the description of many phenomena arising in applied sciences. For instance, many materials can be modeled with sufficient accuracy by using the function spaces $L^{p}$ and $W^{1, p}$, where $p$ is a fixed constant. For some materials with nonhomogeneities, for instance electro-rheological fluids (sometimes referred to as "smart fluids"), this approach is not adequate, but rather the exponent $p$ should be allowed to vary. This leads us to the study of variable exponent Lebesgue and Sobolev spaces, $L^{p(\cdot)}$ and $W^{1, p(\cdot)}$, where $p$ is a real-valued function.

In order to discuss problem $(\mathrm{P})$, we need some results about the space $W_{0}^{1, p(\cdot)}(\Omega)$, which we call variable exponent Sobolev space. We first state some basic properties of $W_{0}^{1, p(\cdot)}(\Omega)$ (for details, see $[12,15,17,25,35,38]$ ). Denote

$$
\begin{aligned}
C_{+}(\bar{\Omega}) & =\{h \mid h \in C(\bar{\Omega}), h(x)>1 \text { for } x \in \bar{\Omega}\}, \\
h^{+} & =\max _{\bar{\Omega}} h(x), h^{-}=\min _{\bar{\Omega}} h(x), \text { for any } h \in C(\bar{\Omega}), \\
L^{p(\cdot)}(\Omega) & =\left\{u \mid u \text { is a measurable real-valued function, } \int_{\Omega}|u(x)|^{p(x)} d x<\infty\right\} .
\end{aligned}
$$

We introduce the norm on $L^{p(\cdot)}(\Omega)$ by

$$
|u|_{p(\cdot)}=\inf \left\{\lambda>\left.0\left|\int_{\Omega}\right| \frac{u(x)}{\lambda}\right|^{p(x)} d x \leq 1\right\} .
$$

Then $\left(L^{p(\cdot)}(\Omega),|\cdot|_{p(\cdot)}\right)$ becomes a Banach space and it is called the variable exponent Lebesgue space.

Proposition 2.1 (see $[12,35])$. i) The space $\left(L^{p(\cdot)}(\Omega),|\cdot|_{p(\cdot)}\right)$ is a separable, uniform convex Banach space, and its conjugate space is $L^{q(\cdot)}(\Omega)$, where $\frac{1}{q(\cdot)}+\frac{1}{p(\cdot)} \equiv$ 1. For any $u \in L^{p(\cdot)}(\Omega)$ and $v \in L^{q(\cdot)}(\Omega)$, we have

$$
\left|\int_{\Omega} u v d x\right| \leq\left(\frac{1}{p^{-}}+\frac{1}{q^{-}}\right)|u|_{p(\cdot)}|v|_{q(\cdot)} .
$$

ii) If $p_{1}, p_{2} \in C_{+}(\bar{\Omega}), p_{1}(x) \leq p_{2}(x)$ for any $x \in \bar{\Omega}$, then $L^{p_{2}(\cdot)}(\Omega) \subset L^{p_{1}(\cdot)}(\Omega)$, and this imbedding is continuous. 
Proposition 2.2 (see $[15,35]$ ). If $f: \Omega \times \mathbb{R} \rightarrow \mathbb{R}$ is a Carathéodory function and satisfies

$$
|f(x, s)| \leq a(x)+b|s|^{p_{1}(x) / p_{2}(x)} \text { for any } x \in \Omega, s \in \mathbb{R},
$$

where $p_{1}, p_{2} \in C_{+}(\bar{\Omega}), a \in L^{p_{2}(\cdot)}(\Omega), a(x) \geq 0, b \geq 0$, then the Nemytsky operator from $L^{p_{1}(\cdot)}(\Omega)$ to $L^{p_{2}(\cdot)}(\Omega)$ defined by $\left(N_{f} u\right)(x)=f(x, u(x))$, is a continuous and bounded operator.

Proposition 2.3 (see $[15,35]$ ). If we denote

$$
\rho(u)=\int_{\Omega}|u|^{p(x)} d x, \forall u \in L^{p(\cdot)}(\Omega),
$$

then there exists $\xi \in \bar{\Omega}$ such that $|u|_{p(\cdot)}^{p(\xi)}=\int_{\Omega}|u|^{p(x)} d x$ and

i) $|u|_{p(\cdot)}<1(=1 ;>1) \Longleftrightarrow \rho(u)<1(=1 ;>1)$;

ii) $|u|_{p(\cdot)}>1 \Longrightarrow|u|_{p(\cdot)}^{p^{-}} \leq \rho(u) \leq|u|_{p(\cdot)}^{p^{+}} ;|u|_{p(\cdot)}<1 \Longrightarrow|u|_{p(\cdot)}^{p^{-}} \geq \rho(u) \geq|u|_{p(\cdot)}^{p^{+}}$;

iii) $|u|_{p(\cdot)} \rightarrow 0 \Longleftrightarrow \rho(u) \rightarrow 0 ;|u|_{p(\cdot)} \rightarrow \infty \Longleftrightarrow \rho(u) \rightarrow \infty$.

Proposition 2.4 (see $[15,35]$ ). If $u, u_{n} \in L^{p(\cdot)}(\Omega), n=1,2, \cdots$, then the following statements are equivalent:

1) $\lim _{k \rightarrow \infty}\left|u_{k}-u\right|_{p(\cdot)}=0$

2) $\lim _{k \rightarrow \infty} \rho\left(u_{k}-u\right)=0$;

3) $u_{k} \rightarrow u$ in measure in $\Omega$ and $\lim _{k \rightarrow \infty} \rho\left(u_{k}\right)=\rho(u)$.

The space $W^{1, p(\cdot)}(\Omega)$ is defined by

$$
W^{1, p(\cdot)}(\Omega)=\left\{u \in L^{p(\cdot)}(\Omega) \mid \nabla u \in\left(L^{p(\cdot)}(\Omega)\right)^{N}\right\},
$$

and it can be equipped with the norm

$$
\|u\|=|u|_{p(\cdot)}+|\nabla u|_{p(\cdot)}, \forall u \in W^{1, p(\cdot)}(\Omega) .
$$

We denote by $W_{0}^{1, p(\cdot)}(\Omega)$ the closure of $C_{0}^{\infty}(\Omega)$ in $W^{1, p(\cdot)}(\Omega)$ and set

$$
p^{*}(x)=\left\{\begin{array}{l}
N p(x) /(N-p(x)), p(x)<N, \\
\infty, p(x) \geq N .
\end{array}\right.
$$

Then we have the following properties.

Proposition 2.5 (see $[12,15,35])$. i) $W^{1, p(\cdot)}(\Omega)$ and $W_{0}^{1, p(\cdot)}(\Omega)$ are separable reflexive Banach spaces;

ii) if $q \in C_{+}(\bar{\Omega})$ and $q(x)<p^{*}(x)$ for any $x \in \bar{\Omega}$, then the imbedding from $W^{1, p(\cdot)}(\Omega)$ to $L^{q(\cdot)}(\Omega)$ is compact;

iii) there is a constant $C>0$ such that

$$
|u|_{p(\cdot)} \leq C|\nabla u|_{p(\cdot)}, \forall u \in W_{0}^{1, p(\cdot)}(\Omega) .
$$


It follows from iii) of Proposition 2.5 that $|\nabla u|_{p(\cdot)}$ and $\|u\|$ are equivalent norms on $W_{0}^{1, p(\cdot)}(\Omega)$. From now on, we will use $|\nabla u|_{p(\cdot)}$ instead of $\|u\|$ as the norm on $W_{0}^{1, p(\cdot)}(\Omega)$.

The Lebesgue and Sobolev spaces with variable exponents coincide with the usual Lebesgue and Sobolev spaces provided that $p$ is constant. These function spaces $L^{p(x)}$ and $W^{1, p(x)}$ have some non-usual properties, see [35, p. 8-9]. Some of these properties are the following:

(i) Assuming that $1<p^{-} \leq p^{+}<\infty$ and $p: \bar{\Omega} \rightarrow[1, \infty)$ is a smooth function, then the following co-area formula

$$
\int_{\Omega}|u(x)|^{p} d x=p \int_{0}^{\infty} t^{p-1}|\{x \in \Omega ;|u(x)|>t\}| d t
$$

has no analogue in the framework of variable exponents.

(ii) Spaces $L^{p(x)}$ do not satisfy the mean continuity property. More exactly, if $p$ is nonconstant and continuous in an open ball $B$, then there is some $u \in L^{p(x)}(B)$ such that $u(x+h) \notin L^{p(x)}(B)$ for every $h \in \mathbb{R}^{N}$ with arbitrary small norm.

(iii) Function spaces with variable exponent are never invariant with respect to translations. The convolution is also limited. For instance, the classical Young inequality

$$
|f * g|_{p(x)} \leq C|f|_{p(x)}\|g\|_{L^{1}}
$$

remains true if and only if $p$ is constant.

Proposition 2.6 (see [16]). If the assumption $\left(\mathrm{p}_{1}\right)$ is satisfied, then $\lambda_{p(\cdot)}$ defined in (1) is positive.

Next, we prove some results related to the $p(x)$-Laplace operator $-\Delta_{p(x)}$ as defined at the beginning of Section 1. Consider the following functional

$$
J(u)=\int_{\Omega} \frac{1}{p(x)}|\nabla u|^{p(x)} d x, u \in X:=W_{0}^{1, p(\cdot)}(\Omega) .
$$

Then (see [9]) $J \in C^{1}(X, \mathbb{R})$ and the $p(x)$-Laplace operator is the derivative operator of $J$ in the weak sense. We denote $L=J^{\prime}: X \rightarrow X^{*}$, then

$$
(L(u), v)=\int_{\Omega}|\nabla u|^{p(x)-2} \nabla u \nabla v d x, \forall v, u \in X .
$$

Theorem 2.7 (see $[15,21])$. i) $L: X \rightarrow X^{*}$ is a continuous, bounded and strictly monotone operator;

ii) $L$ is a mapping of type $\left(S_{+}\right)$, that is, if $u_{n} \rightarrow u$ in $X$ and $\varlimsup_{n \rightarrow+\infty}\left(L\left(u_{n}\right)-\right.$ $\left.L(u), u_{n}-u\right) \leq 0$, then $u_{n} \rightarrow u$ in $X$;

iii) $L: X \rightarrow X^{*}$ is a homeomorphism.

Denote

$$
B\left(x_{0}, \varepsilon, \delta, \theta\right)=\left\{x \in \mathbb{R}^{N}|\delta \leq| x-x_{0} \mid \leq \varepsilon, \frac{x-x_{0}}{\left|x-x_{0}\right|} \cdot \frac{\nabla p\left(x_{0}\right)}{\left|\nabla p\left(x_{0}\right)\right|} \geq \cos \theta\right\},
$$


where $\theta \in\left(0, \frac{\pi}{2}\right)$. Then we obtain the following.

Lemma 2.8. If $p \in C^{1}(\bar{\Omega}), x_{0} \in \Omega$ satisfy $\nabla p\left(x_{0}\right) \neq 0$, then there exists small enough $\varepsilon>0$ such that

$$
\left(x-x_{0}\right) \cdot \nabla p(x)>0, \forall x \in B\left(x_{0}, \varepsilon, \delta, \theta\right),
$$

and

(6) $\max \left\{p(x) \mid x \in \overline{B\left(x_{0}, \varepsilon\right)}\right\}=\max \left\{p(x)\left|x \in B\left(x_{0}, \varepsilon, \delta, \theta\right),\right| x-x_{0} \mid=\varepsilon\right\}$.

Proof. A proof of this lemma can be found in [45]. For readers' convenience, we include it here.

Since $p \in C^{1}(\bar{\Omega})$, for any $x \in B\left(x_{0}, \varepsilon, \delta, \theta\right)$, when $\varepsilon>0$ is small enough, we have

$$
\begin{aligned}
\nabla p(x) \cdot\left(x-x_{0}\right) & =\left(\nabla p\left(x_{0}\right)+o(1)\right) \cdot\left(x-x_{0}\right) \\
& =\nabla p\left(x_{0}\right) \cdot\left(x-x_{0}\right)+o\left(\left|x-x_{0}\right|\right) \\
& \geq\left|\nabla p\left(x_{0}\right)\right|\left|x-x_{0}\right| \cos \theta+o\left(\left|x-x_{0}\right|\right)>0,
\end{aligned}
$$

where $o(1) \in \mathbb{R}^{N}$ is a function and $o(1) \rightarrow 0$ uniformly as $\left|x-x_{0}\right| \rightarrow 0$.

When $\varepsilon$ is small enough, condition (5) is valid. Since $p \in C^{1}(\bar{\Omega})$, there exist a small enough positive $\varepsilon$ such that

$$
p(x)-p\left(x_{0}\right)=\nabla p(y) \cdot\left(x-x_{0}\right)=\left(\nabla p\left(x_{0}\right)+o(1)\right) \cdot\left(x-x_{0}\right),
$$

where $y=x_{0}+\tau\left(x-x_{0}\right)$ and $\tau \in(0,1), o(1) \in \mathbb{R}^{N}$ is a function and $o(1) \rightarrow 0$ uniformly as $\left|x-x_{0}\right| \rightarrow 0$.

Suppose that $x \in \overline{B\left(x_{0}, \varepsilon\right)} \backslash B\left(x_{0}, \varepsilon, \delta, \theta\right)$. Denote $x^{*}=x_{0}+\varepsilon \nabla p\left(x_{0}\right) /\left|\nabla p\left(x_{0}\right)\right|$.

Suppose that $\frac{x-x_{0}}{\left|x-x_{0}\right|} \cdot \frac{\nabla p\left(x_{0}\right)}{\left|\nabla p\left(x_{0}\right)\right|}<\cos \theta$. When $\varepsilon$ is small enough, we have

$$
\begin{aligned}
p(x)-p\left(x_{0}\right) & =\left(\nabla p\left(x_{0}\right)+o(1)\right) \cdot\left(x-x_{0}\right) \\
& <\left|\nabla p\left(x_{0}\right)\right|\left|x-x_{0}\right| \cos \theta+\varepsilon \cdot o(1) \\
& \leq\left(\nabla p\left(x_{0}\right)+o(1)\right) \cdot \varepsilon \nabla p\left(x_{0}\right) /\left|\nabla p\left(x_{0}\right)\right| \\
& =p\left(x^{*}\right)-p\left(x_{0}\right),
\end{aligned}
$$

where $o(1) \in \mathbb{R}^{N}$ is a function and $o(1) \rightarrow 0$ as $\varepsilon \rightarrow 0$.

Suppose that $\left|x-x_{0}\right|<\delta$. When $\varepsilon$ is small enough, we have

$$
\begin{aligned}
p(x)-p\left(x_{0}\right) & =\left(\nabla p\left(x_{0}\right)+o(1)\right) \cdot\left(x-x_{0}\right) \\
& \leq\left|\nabla p\left(x_{0}\right)\right|\left|x-x_{0}\right|+\varepsilon \cdot o(1) \\
& <\left(\nabla p\left(x_{0}\right)+o(1)\right) \cdot \varepsilon \nabla p\left(x_{0}\right) /\left|\nabla p\left(x_{0}\right)\right| \\
& =p\left(x^{*}\right)-p\left(x_{0}\right),
\end{aligned}
$$

where $o(1) \in \mathbb{R}^{N}$ is a function and $o(1) \rightarrow 0$ as $\varepsilon \rightarrow 0$. Thus

$$
\max \left\{p(x) \mid x \in \overline{B\left(x_{0}, \varepsilon\right)}\right\}=\max \left\{p(x) \mid x \in B\left(x_{0}, \varepsilon, \delta, \theta\right)\right\} .
$$

It follows from (5) and (7) that relation (6) holds.

The proof of Lemma 2.8 is thus complete. 
NONHOMOGENEOUS PROBLEMS WITHOUT AMBROSETTI-RABINOWITZ CONDITION 9

Lemma 2.9 Suppose that $F(x, u)$ satisfies $\left(\mathrm{f}_{4}\right)$. Let

$$
h(x)=\left\{\begin{array}{cl}
0, & \text { if }\left|x-x_{0}\right|>\varepsilon \\
\varepsilon-\left|x-x_{0}\right|, & \text { if }\left|x-x_{0}\right| \leq \varepsilon
\end{array},\right.
$$

where $\varepsilon$ is defined as in Lemma 2.8. Then there exists large enough $t$ such that

$$
\int_{\Omega}|\nabla t h|^{p(x)} d x-\int_{\Omega} F(x, t h) d x \rightarrow-\infty \text { as } t \rightarrow+\infty .
$$

Proof. Obviously,

$$
\int_{\Omega} \frac{1}{p(x)}|\nabla t h|^{p(x)} d x \leq C_{2} \int_{B\left(x_{0}, \varepsilon, \delta, \theta\right)}|\nabla t h|^{p(x)} d x .
$$

We make a spherical coordinate transformation. Denote $r=\left|x-x_{0}\right|$. Since $p \in C^{1}(\bar{\Omega})$, it follows from (5) that there exist positive constants $c_{1}$ and $c_{2}$ such that

$$
p(\varepsilon, \omega)-c_{2}(\varepsilon-r) \leq p(r, \omega) \leq p(\varepsilon, \omega)-c_{1}(\varepsilon-r), \forall(r, \omega) \in B\left(x_{0}, \varepsilon, \delta, \theta\right) .
$$

Therefore

$$
\begin{aligned}
\int_{B\left(x_{0}, \varepsilon, \delta, \theta\right)}|\nabla t h|^{p(x)} d x & =\int_{B\left(x_{0}, \varepsilon, \delta, \theta\right)}|t|^{p(r, \omega)} r^{N-1} d r d \omega \\
& \leq \int_{B\left(x_{0}, \varepsilon, \delta, \theta\right)}|t|^{p(\varepsilon, \omega)-c_{1}(\varepsilon-r)} r^{N-1} d r d \omega \\
& \leq \varepsilon^{N-1} \int_{B\left(x_{0}, \varepsilon, \delta, \theta\right)} t^{p(\varepsilon, \omega)-c_{1}(\varepsilon-r)} d r d \omega \\
& \leq \varepsilon^{N-1} \int_{B\left(x_{0}, 1,1, \theta\right)} \frac{t^{p(\varepsilon, \omega)}}{c_{1} \ln t} d \omega .
\end{aligned}
$$

Denote

$$
G(x, u)=\frac{F(x, u)}{|u|^{p(x)}[\ln (e+|u|)]^{p(x)}} .
$$

Then

$$
G(x, u) \rightarrow+\infty \text { uniformly as }|u| \rightarrow+\infty \text { for } x \in \bar{\Omega} \text {. }
$$

Thus there exists a positive constant $M$ such that

$$
G(x, u) \geq 1, \forall|u| \geq M, \forall x \in \bar{\Omega} .
$$

Denote

$$
\begin{aligned}
& E_{1}=\left\{x \in B\left(x_{0}, \varepsilon\right) \mid t h \geq M\right\}=\left\{x \in B\left(x_{0}, \varepsilon\right)|| x-x_{0} \mid \leq \varepsilon-\frac{M}{t}\right\}, \\
& E_{2}=B\left(x_{0}, \varepsilon\right) \backslash E_{1} .
\end{aligned}
$$


Then we have

$$
\begin{aligned}
\int_{\Omega} F(x, t h) d x & =\int_{B\left(x_{0}, \varepsilon\right)} F(x, t h) d x \\
& =\int_{E_{1}} F(x, t h) d x+\int_{E_{2}} F(x, t h) d x \\
& \geq \int_{E_{1}} F(x, t h) d x-C_{1} .
\end{aligned}
$$

When $t$ is large enough, we have

$$
\begin{aligned}
& \int_{E_{1}} F(x, t h) d x \\
& =\int_{E_{1}}|t h|^{p(x)}[\ln (e+|t h|)]^{p(x)} G(x, t h) d x \\
& =\int_{B\left(x_{0}, \varepsilon-\frac{M}{t}, \delta, \theta\right)} C_{1}|t h|^{p(x)}[\ln (e+|t h|)]^{p(x)} G(x, t h) d x \\
& =\int_{B\left(x_{0}, \varepsilon-\frac{M}{t}, \delta, \theta\right)} C_{1}|t(\varepsilon-r)|^{p(r, \omega)} r^{N-1}[\ln (e+|t(\varepsilon-r)|)]^{p(r, \omega)} G(r, \omega, t(\varepsilon-r)) d r d \omega \\
& \geq C_{1} \delta^{N-1} \int_{B\left(x_{0}, \varepsilon-\frac{M}{t}, \delta, \theta\right)}|t|^{p(\varepsilon, \omega)-c_{2}(\varepsilon-r)}|\varepsilon-r|^{p(\varepsilon, \omega)-c_{1}(\varepsilon-r)} \\
& {[\ln (e+|t(\varepsilon-r)|)]^{p(r, \omega)} G(r, \omega, t(\varepsilon-r)) d r d \omega} \\
& =C_{1} \delta^{N-1} \int_{B\left(x_{0}, 1,1, \theta\right)} d \omega \\
& \int_{\delta}^{\varepsilon-\frac{M}{t}}|t|^{p(\varepsilon, \omega)-c_{2}(\varepsilon-r)}|\varepsilon-r|^{p(\varepsilon, \omega)-c_{1}(\varepsilon-r)}[\ln (e+|t(\varepsilon-r)|)]^{p(r, \omega)} G(r, \omega, t(\varepsilon-r)) d r \\
& \geq C_{1} \delta^{N-1} \int_{B\left(x_{0}, 1,1, \theta\right)} d \omega \\
& \int_{\delta}^{\varepsilon-\frac{1}{\ln t}}|t|^{p(\varepsilon, \omega)-c_{2}(\varepsilon-r)}|\varepsilon-r|^{p(\varepsilon, \omega)}[\ln (e+|t(\varepsilon-r)|)]^{p(r, \omega)} G(r, \omega, t(\varepsilon-r)) d r \\
& \geq C_{2} \delta^{N-1} G\left(r_{t}, \omega_{t}, t\left(\varepsilon-r_{t}\right)\right) \\
& \int_{B\left(x_{0}, 1,1, \theta\right)}\left(\frac{1}{\ln t}\right)^{p(\varepsilon, \omega)}\left[\ln \left(e+\frac{t}{\ln t}\right)\right]^{p(\varepsilon, \omega)} \int_{\delta}^{\varepsilon-\frac{1}{\ln t}}|t|^{p(\varepsilon, \omega)-c_{2}(\varepsilon-r)} d r d \omega \\
& \geq C_{3} \delta^{N-1} G\left(r_{t}, \omega_{t}, t\left(\varepsilon-r_{t}\right)\right) \int_{B\left(x_{0}, 1,1, \theta\right)} \frac{|t|^{p(\varepsilon, \omega)-\frac{c_{2}}{\ln t}}}{c_{2} \ln t} d \omega \\
& \geq C_{4} \delta^{N-1} G\left(r_{t}, \omega_{t}, t\left(\varepsilon-r_{t}\right)\right) \int_{B\left(x_{0}, 1,1, \theta\right)} \frac{|t|^{p(\varepsilon, \omega)}}{c_{2} \ln t} d \omega
\end{aligned}
$$

where $\left(r_{t}, \omega_{t}\right) \in E_{1}$ is such that

$$
G\left(r_{t}, \omega_{t}, t\left(\varepsilon-r_{t}\right)\right)=\min \left\{G(r, \omega, t(\varepsilon-r)) \mid(r, \omega) \in B\left(x_{0}, \varepsilon-\frac{1}{\ln t}, \delta, \theta\right)\right\} .
$$


Note that $t\left(\varepsilon-r_{t}\right) \geq \frac{t}{\ln t} \rightarrow+\infty$ as $t \rightarrow+\infty$. Thus

(10) $\int_{\Omega} F(x, t h) d x \geq G\left(r_{t}, \omega_{t}, t\left(\varepsilon-r_{t}\right)\right) C_{5} \int_{B\left(x_{0}, 1,1, \theta\right)} \frac{|t|^{p(\varepsilon, \omega)}}{\ln t} d \omega-C_{1}$ as $t \rightarrow+\infty$.

It follows from (8), (9) and (10) that $\Psi(t h) \rightarrow-\infty$. Proof of Lemma 2.9 is thus complete.

Lemma 2.10 The following $K_{i}(i=1,2,3)$ satisfy the hypothesis $(K)$

$$
\begin{aligned}
& K_{1}(t)=\ln (e+|t|) ; \\
& K_{2}(t)=\ln (e+\ln (e+|t|)) ; \text { and } \\
& K_{3}(t)=[\ln (e+\ln (e+|t|))] \ln (e+|t|) .
\end{aligned}
$$

Proof. We only need to check that $K_{3}(t)$ satisfies the hypothesis $(K)$. The proofs for the other functions are similar.

We observe that $1 \leq K(\cdot) \in C^{1}([0,+\infty),[1,+\infty))$ is increasing and $K(t) \rightarrow+\infty$ as $t \rightarrow+\infty$. So we only need to prove that $t K^{\prime}(t) / K(t) \leq \sigma \in(0,1)$, where $\sigma$ is a constant. By computation we obtain

$$
\begin{aligned}
\frac{t K^{\prime}}{K}= & \frac{t}{K}\left\{\frac{[\ln (e+|t|)] \operatorname{sgn} t}{[e+\ln (e+|t|)](e+|t|)}+\frac{[\ln (e+\ln (e+|t|))] \operatorname{sgn} t}{(e+|t|)}\right\} \\
= & \frac{|t|}{[\ln (e+\ln (e+|t|))][e+\ln (e+|t|)](e+|t|)} \\
& +\frac{|t|}{[\ln (e+|t|)](e+|t|)} .
\end{aligned}
$$

We have

$$
\begin{aligned}
|t| & \leq \frac{1}{3}[\ln (e+\ln (e+|t|))][e+\ln (e+|t|)](e+|t|), \\
|t| & \leq \frac{1}{2}[\ln (e+|t|)](e+|t|)
\end{aligned}
$$

and we complete the proof by observing that

$$
\frac{t K^{\prime}}{K} \leq \frac{5}{6}, \forall t \in \mathbb{R}
$$

\section{Proofs of MAIN RESUlts}

In this section we give the proofs of our main results.

Definition 3.1 We say that $u \in W_{0}^{1, p(\cdot)}(\Omega)$ is a weak solution of $(\mathrm{P})$ if

$$
\int_{\Omega}|\nabla u|^{p(x)-2} \nabla u \cdot \nabla v d x=\int_{\Omega} f(x, u) v d x, \quad \forall v \in X:=W_{0}^{1, p(\cdot)}(\Omega) .
$$

The corresponding functional of $(\mathrm{P})$ is

$$
\varphi(u)=\int_{\Omega} \frac{1}{p(x)}|\nabla u|^{p(x)} d x-\int_{\Omega} F(x, u) d x, \quad \forall u \in X,
$$

where $F(x, t)=\int_{0}^{t} f(x, s) d s$. 
Definition 3.2 We say that $\varphi$ satisfies the Cerami condition in $X$, if any sequence $\left\{u_{n}\right\} \subset X$ such that $\left\{\varphi\left(u_{n}\right)\right\}$ is bounded and $\left\|\varphi^{\prime}\left(u_{n}\right)\right\|\left(1+\left\|u_{n}\right\|\right) \rightarrow 0$ as $n \rightarrow+\infty$ has a convergent subsequence.

Lemma 3.3 If $f$ satisfies $\left(\mathrm{f}_{0}\right)$ and $\left(\mathrm{f}_{1}\right)$, then $\varphi$ satisfies the Cerami condition.

Proof. Let $\left\{u_{n}\right\} \subset X$ be a Cerami sequence, that is $\varphi\left(u_{n}\right) \rightarrow c$ and $\left\|\varphi^{\prime}\left(u_{n}\right)\right\|(1+$ $\left.\left\|u_{n}\right\|\right) \rightarrow 0$. Therefore $\varphi^{\prime}\left(u_{n}\right)=L\left(u_{n}\right)-f\left(x, u_{n}\right) \rightarrow 0$ in $X^{*}$, then we have $L\left(u_{n}\right)=$ $f\left(x, u_{n}\right)+o_{n}(1)$, where $o_{n}(1) \rightarrow 0$ in $X^{*}$ as $n \rightarrow \infty$. Suppose that $\left\{u_{n}\right\}$ is bounded, then $\left\{u_{n}\right\}$ has a weakly convergent subsequence in $X$. Without loss of generality, we assume that $u_{n} \rightarrow u$, then by Proposition 2.2 and 2.5 , we have $f\left(x, u_{n}\right) \rightarrow f(x, u)$ in $X^{*}$. Thus $L\left(u_{n}\right)=f\left(x, u_{n}\right)+o_{n}(1) \rightarrow f(x, u)$ in $X^{*}$. Since $L$ is a homeomorphism, we have $u_{n} \rightarrow L^{-1}(f(x, u))$ in $X$, and so $\varphi$ satisfies the Cerami condition. Therefore $u=L^{-1}(f(x, u))$, then $L(u)=f(x, u)$, this means $u$ is a solution of $(\mathrm{P})$. Thus we only need to prove the boundedness of the Cerami sequence $\left\{u_{n}\right\}$.

We argue by contradiction. Then there exist $c \in \mathbb{R}$ and $\left\{u_{n}\right\} \subset X$ satisfying:

$$
\varphi\left(u_{n}\right) \rightarrow c,\left\|\varphi^{\prime}\left(u_{n}\right)\right\|\left(1+\left\|u_{n}\right\|\right) \rightarrow 0,\left\|u_{n}\right\| \rightarrow+\infty
$$

Obviously,

$$
\left|\frac{1}{p(x)} u_{n}\right|_{p(\cdot)} \leq \frac{1}{p^{-}}\left|u_{n}\right|_{p(\cdot)},\left|\nabla \frac{1}{p(x)} u_{n}\right|_{p(\cdot)} \leq \frac{1}{p^{-}}\left|\nabla u_{n}\right|_{p(\cdot)}+C\left|u_{n}\right|_{p(\cdot)} .
$$

Thus $\left\|\frac{1}{p(x)} u_{n}\right\| \leq C\left\|u_{n}\right\|$. Therefore $\left(\varphi^{\prime}\left(u_{n}\right), \frac{1}{p(x)} u_{n}\right) \rightarrow 0$. We may assume that

$$
\begin{aligned}
c+1 \geq & \varphi\left(u_{n}\right)-\left(\varphi^{\prime}\left(u_{n}\right), \frac{1}{p(x)} u_{n}\right) \\
= & \int_{\Omega} \frac{1}{p(x)}\left|\nabla u_{n}\right|^{p(x)} d x-\int_{\Omega} F\left(x, u_{n}\right) d x \\
& -\left\{\int_{\Omega} \frac{1}{p(x)}\left|\nabla u_{n}\right|^{p(x)} d x-\int_{\Omega} \frac{1}{p(x)} f\left(x, u_{n}\right) u_{n} d x-\right. \\
& \left.\int_{\Omega} \frac{1}{p^{2}(x)} u_{n}\left|\nabla u_{n}\right|^{p(x)-2} \nabla u_{n} \nabla p d x\right\} \\
\geq & \int_{\Omega} \frac{1}{p^{2}(x)} u_{n}\left|\nabla u_{n}\right|^{p(x)-2} \nabla u_{n} \nabla p d x+\int_{\Omega}\left\{\frac{1}{p(x)} f\left(x, u_{n}\right) u_{n}-F\left(x, u_{n}\right)\right\} d x .
\end{aligned}
$$

Hence

$$
\begin{aligned}
\int_{\Omega}\left\{\frac{f\left(x, u_{n}\right) u_{n}}{p(x)}-F\left(x, u_{n}\right)\right\} d x \leq & C_{0}\left(\int_{\Omega}\left|u_{n}\right|\left|\nabla u_{n}\right|^{p(x)-1} d x+1\right) \\
\leq & \sigma \int_{\Omega} \frac{\left|\nabla u_{n}\right|^{p(x)}}{K\left(\left|u_{n}\right|\right)} d x+C_{1} \\
& +C(\sigma) \int_{\Omega}\left|u_{n}\right|^{p(x)}\left[K\left(\left|u_{n}\right|\right)\right]^{p(x)-1} d x
\end{aligned}
$$

where $\sigma$ is a small enough positive constant. 
Due to hypothesis $(K)$, it is easy to check that $\frac{u_{n}}{K\left(\left|u_{n}\right|\right)} \in X$, and $\left\|\frac{u_{n}}{K\left(\left|u_{n}\right|\right)}\right\| \leq$ $C_{2}\left\|u_{n}\right\|$. Let $\frac{u_{n}}{K\left(\left|u_{n}\right|\right)}$ be a test function. We have

$$
\begin{aligned}
& \int_{\Omega} f\left(x, u_{n}\right) \frac{u_{n}}{K\left(\left|u_{n}\right|\right)} d x \\
= & \int_{\Omega}\left|\nabla u_{n}\right|^{p(x)-2} \nabla u_{n} \nabla \frac{u_{n}}{K\left(\left|u_{n}\right|\right)} d x+o(1) \\
= & \int_{\Omega} \frac{\left|\nabla u_{n}\right|^{p(x)}}{K\left(\left|u_{n}\right|\right)} d x-\int_{\Omega} u_{n}\left|\nabla u_{n}\right|^{p(x)-2} \nabla u_{n} \nabla \frac{1}{K\left(\left|u_{n}\right|\right)} d x+o(1) .
\end{aligned}
$$

By computation, we obtain

$$
\begin{aligned}
& \left.\left|\int_{\Omega} u_{n}\right| \nabla u_{n}\right|^{p(x)-2} \nabla u_{n} \nabla \frac{1}{K\left(\left|u_{n}\right|\right)} d x \mid \\
\leq & \int_{\Omega}\left|u_{n}\right|\left|\nabla u_{n}\right|^{p(x)-1} \frac{\left|\nabla K\left(\left|u_{n}\right|\right)\right|}{K^{2}\left(\left|u_{n}\right|\right)} d x \\
\leq & \int_{\Omega} \frac{\left|\nabla u_{n}\right|^{p(x)}}{K\left(\left|u_{n}\right|\right)} \frac{\left|u_{n}\right| K^{\prime}\left(\left|u_{n}\right|\right)}{K\left(\left|u_{n}\right|\right)} d x .
\end{aligned}
$$

Note that $\frac{\left|u_{n}\right| K^{\prime}\left(\left|u_{n}\right|\right)}{K\left(\left|u_{n}\right|\right)} \leq \sigma_{0} \in(0,1)$. Thus

$$
C_{3} \int_{\Omega} \frac{\left|\nabla u_{n}\right|^{p(x)}}{K\left(\left|u_{n}\right|\right)} d x-C_{4} \leq \int_{\Omega} \frac{f\left(x, u_{n}\right) u_{n}}{K\left(\left|u_{n}\right|\right)} d x \leq C_{5} \int_{\Omega} \frac{\left|\nabla u_{n}\right|^{p(x)}}{K\left(\left|u_{n}\right|\right)} d x+C_{6} .
$$

By (11), (12) and conditions $\left(\mathrm{f}_{0}\right)$ and $\left(\mathrm{f}_{1}\right)$, we have

$$
\begin{aligned}
& \int_{\Omega} f\left(x, u_{n}\right) \frac{u_{n}}{K\left(\left|u_{n}\right|\right)} d x \\
& \stackrel{\left(\mathrm{f}_{1}\right)}{\leq} C_{7} \int_{\Omega}\left\{\frac{f\left(x, u_{n}\right) u_{n}}{p(x)}-F\left(x, u_{n}\right)\right\} d x+C_{7} \\
\leq & C_{7}\left\{\sigma \int_{\Omega} \frac{\left|\nabla u_{n}\right|^{p(x)}}{K\left(\left|u_{n}\right|\right)} d x+C_{8}+C(\sigma) \int_{\Omega}\left|u_{n}\right|^{p(x)}\left[K\left(\left|u_{n}\right|\right)\right]^{p(x)-1} d x\right\} \\
\leq & C_{7} \sigma \int_{\Omega} \frac{\left|\nabla u_{n}\right|^{p(x)}}{K\left(\left|u_{n}\right|\right)} d x+C_{7} C(\sigma) \int_{\Omega}\left|u_{n}\right|^{p(x)}\left[K\left(\left|u_{n}\right|\right)\right]^{p(x)-1} d x+C_{9} \\
& \stackrel{(12)}{\leq} \frac{1}{2} \int_{\Omega} \frac{f\left(x, u_{n}\right) u_{n}}{K\left(\left|u_{n}\right|\right)} d x+C_{7} C(\sigma) \int_{\Omega}\left|u_{n}\right|^{p(x)}\left[K\left(\left|u_{n}\right|\right)\right]^{p(x)-1} d x+C_{10} .
\end{aligned}
$$

Thus, by condition $\left(\mathrm{f}_{1}\right)$ and the above inequality, we can see

$$
\begin{aligned}
& \int_{\Omega} f\left(x, u_{n}\right) \frac{u_{n}}{K\left(\left|u_{n}\right|\right)} d x \\
\leq & C_{11} \int_{\Omega}\left|u_{n}\right|^{p(x)}\left[K\left(\left|u_{n}\right|\right)\right]^{p(x)-1} d x+C_{12} .
\end{aligned}
$$

Note that $\frac{t f(x, t)}{|t|^{p(x)}[K(t)]^{p(x)}} \rightarrow+\infty$ uniformly as $|t| \rightarrow+\infty$ for $x \in \bar{\Omega}$. We claim that

$$
\int_{\Omega}\left|u_{n}\right|^{p(x)}\left[K\left(\left|u_{n}\right|\right)\right]^{p(x)-1} d x \quad \text { is bounded. }
$$


This means that

$$
\int_{\Omega} f\left(x, u_{n}\right) \frac{u_{n}}{K\left(\left|u_{n}\right|\right)} d x \text { is bounded. }
$$

In fact, by $(\mathrm{K})$, we observe that there exists $M>0$ large enough such that

$$
\frac{t f(x, t)}{K(t)}>2 C_{11}|t|^{p(x)}[K(t)]^{p(x)-1}, \forall|t| \geq M .
$$

Denote $\Omega_{n}=\left\{x \in \Omega|| u_{n} \mid \geq M\right\}$. We have

$$
\int_{\Omega} f\left(x, u_{n}\right) \frac{u_{n}}{K\left(\left|u_{n}\right|\right)} d x \geq \int_{\Omega_{n}} 2 C_{11}\left|u_{n}\right|^{p(x)}\left[K\left(\left|u_{n}\right|\right)\right]^{p(x)-1} d x-C_{12} .
$$

Combining (13)-(15), we obtain

$$
\int_{\Omega_{n}} C_{11}\left|u_{n}\right|^{p(x)}\left[K\left(\left|u_{n}\right|\right)\right]^{p(x)-1} d x \leq C_{13},
$$

and hence

$$
\int_{\Omega} C_{11}\left|u_{n}\right|^{p(x)}\left[K\left(\left|u_{n}\right|\right)\right]^{p(x)-1} d x \leq C_{14}
$$

Thus

$$
\int_{\Omega} f\left(x, u_{n}\right) \frac{u_{n}}{K\left(\left|u_{n}\right|\right)} d x \leq C_{14}, \text { for any } n=1,2, \cdots
$$

This combine $\left(f_{0}\right)$ implies that

$$
\left\{\int_{\Omega} \frac{\left|f\left(x, u_{n}\right) u_{n}\right|}{K\left(\left|u_{n}\right|\right)} d x\right\} \text { is bounded. }
$$

Let $\varepsilon>0$ satisfy $\varepsilon<\min \left\{1, p^{-}-1, \frac{1}{p^{*+}},\left(\frac{p^{*}}{\alpha}\right)^{-}-1\right\}$. Since $\left\|\varphi^{\prime}\left(u_{n}\right)\right\|\left\|u_{n}\right\| \rightarrow 0$, we get

$$
\begin{aligned}
\int_{\Omega}\left|\nabla u_{n}\right|^{p(x)} d x & =\int_{\Omega} f\left(x, u_{n}\right) u_{n} d x+o(1) \\
& \leq \int_{\Omega}^{\varepsilon}\left|f\left(x, u_{n}\right) u_{n}\right|^{\varepsilon}\left[K\left(\left|u_{n}\right|\right)\right]^{1-\varepsilon}\left[\frac{\left|f\left(x, u_{n}\right) u_{n}\right|}{K\left(\left|u_{n}\right|\right)}\right]^{1-\varepsilon} d x+o(1) .
\end{aligned}
$$

By condition $\left(\mathrm{f}_{1}\right)$, we have

$$
\left|f\left(x, u_{n}\right) u_{n}\right| \geq\left|u_{n}\right|^{p(x)} \text { for large enough }\left|u_{n}\right|,
$$

and

$$
\left[K\left(\left|u_{n}\right|\right)\right]^{1-\varepsilon} \leq\left[\ln \left(e+\left|u_{n}\right|\right)\right]^{2(1-\varepsilon)} \text { for large enough }\left|u_{n}\right|,
$$

then we have

$$
\left|f\left(x, u_{n}\right) u_{n}\right|^{\varepsilon}\left[K\left(\left|u_{n}\right|\right)\right]^{1-\varepsilon} \leq C_{15}\left(\left|f\left(x, u_{n}\right) u_{n}\right|^{\varepsilon(1+\varepsilon)}+1\right) .
$$

Therefore

$$
\begin{aligned}
\int_{\Omega}\left|\nabla u_{n}\right|^{p(x)} d x & =\int_{\Omega} f\left(x, u_{n}\right) u_{n} d x+o(1) \\
& \leq C_{15}\left(1+\left\|u_{n}\right\|\right)^{1+\varepsilon} \int_{\Omega}\left[\frac{\left|f\left(x, u_{n}\right) u_{n}\right|^{1+\varepsilon}+1}{\left(1+\left\|u_{n}\right\|\right)^{\frac{1+\varepsilon}{\varepsilon}}}\right]^{\varepsilon}\left[\frac{\left|f\left(x, u_{n}\right) u_{n}\right|}{K\left(\left|u_{n}\right|\right)}\right]^{1-\varepsilon} d x+o(1) .
\end{aligned}
$$


By Young's inequality, we have

$$
\int_{\Omega}\left|\nabla u_{n}\right|^{p(x)} d x \leq C_{15}\left(1+\left\|u_{n}\right\|\right)^{1+\varepsilon} \int_{\Omega} \frac{\left|f\left(x, u_{n}\right) u_{n}\right|^{1+\varepsilon}+1}{\left(1+\| u_{n}||\right)^{\frac{1+\varepsilon}{\varepsilon}}}+\frac{\left|f\left(x, u_{n}\right) u_{n}\right|}{K\left(\left|u_{n}\right|\right)} d x+o(1) .
$$

According to the definition of $\varepsilon$, we have

$$
\left|f\left(x, u_{n}\right) u_{n}\right|^{1+\varepsilon}+1 \leq C\left(\left|u_{n}\right|^{p^{*}(x)}+1\right)
$$

and

$$
\left(1+\left\|u_{n}\right\|\right)^{\frac{1+\varepsilon}{\varepsilon}} \geq\left(1+\left\|u_{n}\right\|\right)^{(1+\varepsilon)\left(p^{*}\right)^{+}} .
$$

Therefore

Thus, the sequence

$$
\begin{gathered}
\int_{\Omega} \frac{\left|f\left(x, u_{n}\right) u_{n}\right|^{1+\varepsilon}+1}{\left(1+\left\|u_{n}\right\|\right)^{\frac{1+\varepsilon}{\varepsilon}}} d x \leq \int_{\Omega} \frac{C\left(\left|u_{n}\right|^{p^{*}(x)}+1\right)}{\left(1+\left\|u_{n}\right\|\right)^{\frac{1+\varepsilon}{\varepsilon}}} d x \leq \\
\leq \frac{C\left(\left|u_{n}\right|^{\left(p^{*}\right)^{+}}+1\right)}{\left(1+\left\|u_{n}\right\|\right)^{\frac{1+\varepsilon}{\varepsilon}}} \leq \frac{C_{\#}\left(\left\|u_{n}\right\|^{\left(p^{*}\right)^{+}}+1\right)}{\left(1+\left\|u_{n}\right\|\right)^{\frac{1+\varepsilon}{\varepsilon}}} .
\end{gathered}
$$

$$
\left\{\int_{\Omega} \frac{\left|f\left(x, u_{n}\right) u_{n}\right|^{1+\varepsilon}+1}{\left(1+\left\|u_{n}\right\|\right)^{\frac{1+\varepsilon}{\varepsilon}}} d x\right\}
$$

is bounded. This combine (16) and (17) implies

$$
\int_{\Omega}\left|\nabla u_{n}\right|^{p(x)} d x \leq C_{16}\left(1+\left\|u_{n}\right\|\right)^{1+\varepsilon}+C_{17} .
$$

Note that $\varepsilon<p^{-}-1$. This is a contradiction, hence $\left\{u_{n}\right\}$ is bounded in $X$.

The proof of Lemma 3.3 is thus complete.

Proof of Theorem 1.1. We first establish the existence of a nontrivial weak solution.

We show that $\varphi$ satisfies conditions of the mountain pass lemma. By Lemma 3.3, $\varphi$ satisfies the Cerami condition. Since $p(x)<\alpha(x)<p^{*}(x)$, the embedding $X \hookrightarrow L^{\alpha(\cdot)}(\Omega)$ is compact. Hence there exists $C_{0}>0$ such that

$$
|u|_{p(\cdot)} \leq C_{0}\|u\|, \forall u \in X .
$$

Let $\sigma>0$ be small enough such that $\sigma \leq \frac{1}{4} \lambda_{p(\cdot)}$. By the assumptions $\left(\mathrm{f}_{0}\right)$ and $\left(\mathrm{f}_{2}\right)$, we obtain

$$
F(x, t) \leq \sigma \frac{1}{p(x)}|t|^{p(x)}+C(\sigma)|t|^{\alpha(x)}, \forall(x, t) \in \Omega \times \mathbb{R} .
$$

By $\left(\mathrm{p}_{1}\right)$ and Lemma 2.6, we have $\lambda_{p(\cdot)}>0$ and

$$
\int_{\Omega} \frac{1}{p(x)}|\nabla u|^{p(x)} d x-\sigma \int_{\Omega} \frac{1}{p(x)}|u|^{p(x)} d x \geq \frac{3}{4} \int_{\Omega} \frac{1}{p(x)}|\nabla u|^{p(x)} .
$$

Since $\alpha \in C(\bar{\Omega})$ and $p(x)<\alpha(x)<p^{*}(x)$, we can divide the domain $\Omega$ into $n_{0}$ disjoint small subdomains $\Omega_{i}\left(i=1, \cdots, n_{0}\right)$ such that $\bar{\Omega}=\bigcup_{i=1}^{n_{0}} \overline{\Omega_{i}}$ and

$$
\sup _{\Omega_{i}} p(x)<\inf _{\Omega_{i}} \alpha(x) \leq \sup _{\Omega_{i}} \alpha(x)<\inf _{\Omega_{i}} p^{*}(x) .
$$


Let

$$
\epsilon=\min _{1 \leq i \leq n_{0}}\left\{\inf _{\Omega_{i}} \alpha(x)-\sup _{\Omega_{i}} p(x)\right\} .
$$

and denote by $\|u\|_{\Omega_{i}}$ the norm of $u$ on $\Omega_{i}$, that is

$$
\int_{\Omega_{i}} \frac{1}{p(x)}\left|\nabla \frac{u}{\|u\|_{\Omega_{i}}}\right|^{p(x)} d x+\int_{\Omega_{i}} \frac{1}{p(x)}\left|\frac{u}{\|u\|_{\Omega_{i}}}\right|^{p(x)} d x=1 .
$$

Then $\|u\|_{\Omega_{i}} \leq C\|u\|$ and there exist $\xi_{i}, \eta_{i} \in \overline{\Omega_{i}}$ such that

$$
\begin{aligned}
|u|_{\alpha(\cdot)}^{\alpha\left(\xi_{i}\right)} & =\int_{\Omega_{i}}|u|^{\alpha(x)} d x \\
\|u\|_{\Omega_{i}}^{p\left(\eta_{i}\right)} & =\int_{\Omega_{i}}\left(\frac{1}{p(x)}|\nabla u|^{p(x)}+\frac{1}{p(x)}|u|^{p(x)}\right) d x .
\end{aligned}
$$

When $\|u\|$ is small enough, we have

$$
\begin{aligned}
C(\sigma) \int_{\Omega}|u|^{\alpha(x)} d x & =C(\sigma) \sum_{i=1}^{n_{0}} \int_{\Omega_{i}}|u|^{\alpha(x)} d x \\
& \left.=C(\sigma) \sum_{i=1}^{n_{0}}|u|_{\alpha(\cdot)}^{\alpha\left(\xi_{i}\right)} \text { (where } \xi_{i} \in \overline{\Omega_{i}}\right) \\
& \left.\leq C \sum_{i=1}^{n_{0}}\|u\|_{\Omega_{i}}^{\alpha\left(\xi_{i}\right)} \text { (by Proposition } 2.5\right) \\
& \left.\leq C\|u\|^{\epsilon} \sum_{i=1}^{n_{0}}\|u\|_{\Omega_{i}}^{p\left(\eta_{i}\right)} \text { (where } \eta_{i} \in \overline{\Omega_{i}}\right) \\
& =C\|u\|^{\epsilon} \sum_{i=1}^{n_{0}} \int_{\Omega_{i}}\left(\frac{1}{p(x)}|\nabla u|^{p(x)}+\frac{1}{p(x)}|u|^{p(x)}\right) d x \\
& =C\|u\|^{\epsilon} \int_{\Omega}\left(\frac{1}{p(x)}|\nabla u|^{p(x)}+\frac{1}{p(x)}|u|^{p(x)}\right) d x \\
& \leq \frac{1}{4} \int_{\Omega} \frac{1}{p(x)}|\nabla u|^{p(x)} d x .
\end{aligned}
$$

Thus

$$
\begin{aligned}
\varphi(u) & \geq \int_{\Omega} \frac{1}{p(x)}|\nabla u|^{p(x)}-\sigma \int_{\Omega} \frac{1}{p(x)}|u|^{p(x)} d x-C(\sigma) \int_{\Omega}|u|^{\alpha(x)} d x \\
& \geq \frac{1}{2} \int_{\Omega} \frac{1}{p(x)}|\nabla u|^{p(x)} \text { when }\|u\| \text { is small enough. }
\end{aligned}
$$

Therefore, there exist $r>0$ and $\delta>0$ such that $\varphi(u) \geq \delta>0$ for every $u \in X$ and $\|u\|=r$.

Suppose $\left(\mathrm{p}_{2}\right)$ is satisfied. Define $h \in C_{0} \overline{\left(B\left(x_{0}, 3 \delta\right)\right)}$ as follows:

$$
h(x)=\left\{\begin{array}{cc}
0, & \left|x-x_{0}\right| \geq 3 \delta \\
3 \delta-\left|x-x_{0}\right|, & 2 \delta \leq\left|x-x_{0}\right|<3 \delta \\
\delta, & \left|x-x_{0}\right|<2 \delta
\end{array} .\right.
$$

Note that

$$
\min _{\left|x-x_{0}\right| \leq \delta} p(x)>\max _{2 \delta \leq\left|x-x_{0}\right| \leq 3 \delta} p(x) .
$$


It is now easy to check that

$$
\begin{aligned}
\varphi(t h)= & \int_{\Omega} \frac{1}{p(x)}|\nabla t h|^{p(x)}-\int_{\Omega} F(x, t h) d x \leq \int_{\overline{B\left(x_{0}, 3 \delta\right)} \backslash\left(\overline{B\left(x_{0}, 2 \delta\right)}\right)} \frac{1}{p(x)}|\nabla t h|^{p(x)}- \\
& \int_{\left(\overline{B\left(x_{0}, \delta\right)}\right)} C_{1}|t h|^{p(x)} d x+C_{2} \rightarrow-\infty \text { as } t \rightarrow+\infty .
\end{aligned}
$$

Since $\varphi(0)=0, \varphi$ satisfies the conditions of the mountain pass lemma. So $\varphi$ admits at least one nontrivial critical point, which implies the problem $(\mathrm{P})$ has a nontrivial weak solution $u$.

Suppose $\left(\mathrm{f}_{4}\right)$ is satisfied. We may assume that there exists $x_{0} \in \Omega$ such that $\nabla p\left(x_{0}\right) \neq 0$.

Define $h \in C_{0}\left(\overline{B\left(x_{0}, \varepsilon\right)}\right)$ as follows:

$$
h(x)=\left\{\begin{array}{cl}
0, & \left|x-x_{0}\right| \geq \varepsilon \\
\varepsilon-\left|x-x_{0}\right|, & \left|x-x_{0}\right|<\varepsilon
\end{array} .\right.
$$

By $\left(\mathrm{f}_{4}\right)$ and Lemma 2.9, there exists $\varepsilon>0$ small enough such that

$$
\varphi(t h)=\int_{\Omega} \frac{1}{p(x)}|\nabla t h|^{p(x)}-\int_{\Omega} F(x, t h) d x \rightarrow-\infty \text { as } t \rightarrow+\infty .
$$

Since $\varphi(0)=0, \varphi$ satisfies the conditions of the mountain pass lemma. So $\varphi$ admits at least one nontrivial critical point, which implies that problem $(\mathrm{P})$ has a nontrivial weak solution $u$. The proof of Theorem 1.1 is thus complete.

In order to prove Theorem 1.2, we need to do some preparations. Note that $X:=W_{0}^{1, p(\cdot)}(\Omega)$ is a reflexive and separable Banach space (see [47], Section 17, Theorem 2-3). Therefore there exist $\left\{e_{j}\right\} \subset X$ and $\left\{e_{j}^{*}\right\} \subset X^{*}$ such that

$$
X=\overline{\operatorname{span}}\left\{e_{j}, j=1,2, \cdots\right\}, \quad X^{*}=\overline{\operatorname{span}}^{W^{*}}\left\{e_{j}^{*}, j=1,2, \cdots\right\},
$$

and

$$
<e_{j}^{*}, e_{j}>= \begin{cases}1, & \text { if } i=j \\ 0, & \text { if } i \neq j\end{cases}
$$

For convenience, we write $X_{j}=\operatorname{span}\left\{e_{j}\right\}, Y_{k}=\bigoplus_{j=1}^{k} X_{j}$ and $Z_{k}=\overline{\bigoplus_{j=k}^{\infty} X_{j}}$.

Lemma 3.4. Assume that $\alpha \in C_{+}(\bar{\Omega}), \alpha(x)<p^{*}(x)$ for any $x \in \bar{\Omega}$. If

$$
\beta_{k}=\sup \left\{|u|_{\alpha(\cdot)} \mid\|u\|=1, u \in Z_{k}\right\}
$$

then $\lim _{k \rightarrow \infty} \beta_{k}=0$.

Proof. Obviously, $0<\beta_{k+1} \leq \beta_{k}$, so $\beta_{k} \rightarrow \beta \geq 0$. Let $u_{k} \in Z_{k}$ satisfy

$$
\left\|u_{k}\right\|=1,0 \leq \beta_{k}-\left|u_{k}\right|_{\alpha(\cdot)}<\frac{1}{k} \text {. }
$$

Then there exists a subsequence of $\left\{u_{k}\right\}$ (which we still denote by $u_{k}$ ) such that $u_{k} \rightarrow u$, and

$$
<e_{j}^{*}, u>=\lim _{k \rightarrow \infty}\left\langle e_{j}^{*}, u_{k}\right\rangle=0, \forall e_{j}^{*}
$$


This implies that $u=0$, and so $u_{k} \rightarrow 0$. Since the embedding from $W_{0}^{1, p(\cdot)}(\Omega)$ into $L^{\alpha(\cdot)}(\Omega)$ is compact, we can conclude that $u_{k} \rightarrow 0$ in $L^{\alpha(\cdot)}(\Omega)$. Hence we get $\beta_{k} \rightarrow 0$ as $k \rightarrow \infty$. The proof of Lemma 3.4 is thus complete.

In order to prove Theorem 1.2, we need the following auxiliary result, see [50, Theorem 4.7]. If the Cerami condition is replaced by PS condition, we can use the following property, see [9, Theorem 3.6].

Lemma 3.5. Suppose that $\varphi \in C^{1}(X, \mathbb{R})$ is even and satisfies the Cerami condition. Let $V^{+}, V^{-} \subset X$ be closed subspaces of $X$ with codim $V^{+}+1=\operatorname{dim}$ $V^{-}$. Suppose that:

$\left(1^{0}\right) \varphi(0)=0$

$\left(2^{0}\right) \exists \tau>0, \gamma>0$ such that $\forall u \in V^{+}:\|u\|=\gamma \Rightarrow \varphi(u) \geq \tau$; and

$\left(3^{0}\right) \exists \rho>0$ such that $\forall u \in V^{-}:\|u\| \geq \rho \Rightarrow \varphi(u) \leq 0$.

Consider the following set:

$$
\Gamma=\left\{g \in C^{0}(X, X) \mid g \text { is odd, } g(u)=u \text { if } u \in V^{-} \text {and }\|u\| \geq \rho\right\} \text {. }
$$

Then

(a) $\forall \delta>0, g \in \Gamma, S_{\delta}^{+} \cap g\left(V^{-}\right) \neq \emptyset$, here $S_{\delta}^{+}=\left\{u \in V^{+} \mid\|u\|=\delta\right\}$; and

(b) the number $\varpi:=\inf _{g \in \Gamma} \sup _{u \in V^{-}} \varphi(g(u)) \geq \tau>0$ is a critical value for $\varphi$.

Proof of Theorem 1.2. We first establish the existence of infinitely many pairs of weak solutions.

According to $\left(f_{0}\right),\left(f_{1}\right)$ and $\left(f_{3}\right), \varphi$ is an even functional and satisfies the Cerami condition. Let $V_{k}^{+}=Z_{k}$ be a closed linear subspace of $X$ and $V_{k}^{+} \oplus Y_{k-1}=X$.

Suppose that $\left(\mathrm{f}_{4}\right)$ is satisfied. We may assume that there exists $x_{n} \in \Omega$ such that $\nabla p\left(x_{n}\right) \neq 0$.

Define $h_{n} \in C_{0}\left(\overline{B\left(x_{n}, \varepsilon_{n}\right)}\right)$ by

$$
h_{n}(x)=\left\{\begin{array}{cc}
0, & \left|x-x_{n}\right| \geq \varepsilon_{n} \\
\varepsilon_{n}-\left|x-x_{n}\right|, & \left|x-x_{n}\right|<\varepsilon_{n}
\end{array} .\right.
$$

Without loss of generality, we may assume that

$$
\operatorname{supp} h_{i} \cap \operatorname{supp} h_{j}=\emptyset, \forall i \neq j \text {. }
$$

By Lemma 2.9, we can let $\varepsilon_{n}>0$ be small enough so that

$$
\varphi\left(t h_{n}\right)=\int_{\Omega} \frac{1}{p(x)}\left|\nabla t h_{n}\right|^{p(x)}-\int_{\Omega} F\left(x, t h_{n}\right) d x \rightarrow-\infty \text { as } t \rightarrow+\infty .
$$

Suppose that $\left(\mathrm{p}_{3}\right)$ is satisfied. Define $h_{n} \in C_{0}\left(\overline{B\left(x_{n}, \varepsilon_{n}\right)}\right)$ by

$$
h_{n}(x)=\left\{\begin{array}{cc}
0, & \left|x-x_{n}\right| \geq 3 \delta_{n} \\
3 \delta_{n}-\left|x-x_{n}\right|, & 2 \delta_{n} \leq\left|x-x_{n}\right|<3 \delta_{n} \\
\delta_{n}, & \left|x-x_{n}\right|<2 \delta_{n}
\end{array} .\right.
$$


Note that $\min _{\left|x-x_{n}\right| \leq \delta_{n}} p(x)>\max _{2 \delta_{n} \leq\left|x-x_{n}\right| \leq 3 \delta_{n}} p(x)$. It follows that

$$
\begin{aligned}
\varphi\left(t h_{n}\right) & =\int_{\Omega} \frac{1}{p(x)}\left|\nabla t h_{n}\right|^{p(x)}-\int_{\Omega} F\left(x, t h_{n}\right) d x \\
& \leq \int_{2 \delta_{n} \leq\left|x-x_{n}\right| \leq 3 \delta_{n}} \frac{1}{p(x)}\left|\nabla t h_{n}\right|^{p(x)}-\int_{\left|x-x_{n}\right| \leq \delta_{n}} C_{1}\left|t h_{n}\right|^{p(x)} d x+C_{2} \rightarrow-\infty
\end{aligned}
$$

as $t \rightarrow+\infty$.

Set $V_{k}^{-}=\operatorname{span}\left\{h_{1}, \cdots, h_{k}\right\}$. We will prove that there exist infinitely many pairs of $V_{k}^{+}$and $V_{k}^{-}$, such that $\varphi$ satisfies the conditions of Lemma 3.5 and the corresponding critical value satisfies

$$
\varpi_{k}:=\inf _{g \in \Gamma} \sup _{u \in V_{k}^{-}} \varphi(g(u)) \rightarrow+\infty
$$

when $k \rightarrow+\infty$. This shows that there are infinitely many pairs of solutions of the problem $(\mathrm{P})$.

For any $m=1,2, \cdots$, we will prove that there exist $\rho_{m}>\gamma_{m}>0$ and large enough $k_{m}$ such that

$$
\begin{array}{ll}
\left(A_{1}\right) \quad b_{k_{m}}: & =\inf \left\{\varphi(u) \mid u \in V_{k_{m}}^{+},\|u\|=\gamma_{m}\right\} \rightarrow+\infty(m \rightarrow+\infty) ; \text { and } \\
\left(A_{2}\right) \quad a_{k_{m}}: & =\max \left\{\varphi(u) \mid u \in V_{k_{m}}^{-},\|u\|=\rho_{m}\right\} \leq 0 .
\end{array}
$$

First, we prove $\left(A_{1}\right)$ as follows. By computation, for any $u \in Z_{k_{m}}$ with $\|u\|=$ $\gamma_{m}=m$, we have

$$
\begin{aligned}
\varphi(u) & =\int_{\Omega} \frac{1}{p(x)}|\nabla u|^{p(x)} d x-\int_{\Omega} F(x, u) d x \\
& \geq \frac{1}{p^{+}} \int_{\Omega}|\nabla u|^{p(x)} d x-C \int_{\Omega}|u|^{\alpha(x)} d x-C_{1} \int_{\Omega}|u| d x \\
& \geq \frac{1}{p^{+}}\|u\|^{p^{-}}-C|u|_{\alpha(\cdot)}^{\alpha(\xi)}-C_{2}|u|_{\alpha(\cdot)} \quad(\text { where } \xi \in \Omega) \\
& \geq\left\{\begin{array}{l}
\frac{1}{p^{+}}\|u\|^{p^{-}}-C \beta_{k_{m}}^{\alpha^{-}}\|u\|^{\alpha^{-}}-C_{2} \beta_{k_{m}}\|u\|, \text { if }|u|_{\alpha(\cdot)} \leq 1, \\
\frac{1}{p^{+}}\|u\|^{p^{-}}-C \beta_{k_{m}}^{\alpha^{+}}\|u\|^{\alpha^{+}}-C_{2} \beta_{k_{m}}\|u\|, \text { if }|u|_{\alpha(\cdot)}>1, \\
\geq \frac{1}{p^{+}}\|u\|^{p^{-}}-C \beta_{k_{m}}^{\alpha^{-}}\left(\|u\|^{\alpha^{+}}+1\right)-C_{2} \beta_{k_{m}}\|u\| .
\end{array}\right.
\end{aligned}
$$

Obviously, there exists a large enough $k_{m}$ such that

$\frac{1}{p^{+}}\|u\|^{p^{-}}-C \beta_{k_{m}}^{\alpha^{-}}\left(\|u\|^{\alpha^{+}}+1\right)-C_{2} \beta_{k_{m}}\|u\| \geq \frac{1}{2 p^{+}}\|u\|^{p^{-}}, \forall u \in Z_{k_{m}}$ with $\|u\|=\gamma_{m}=m$.

Therefore $\varphi(u) \geq \frac{1}{2 p^{+}}\|u\|^{p^{-}}, \forall u \in Z_{k_{m}}$ with $\|u\|=\gamma_{m}=m$. Hence $b_{k_{m}} \rightarrow+\infty$ as $m \rightarrow \infty$.

Now we give a proof of $\left(A_{2}\right)$. According to the above discussion, it is easy to see that $\Psi\left(t h_{k_{m}}\right) \rightarrow-\infty$ as $t \rightarrow+\infty$. Therefore

$$
\varphi(t h) \rightarrow-\infty \text { as } t \rightarrow+\infty, \forall h \in V_{k_{m}}^{-}=\operatorname{span}\left\{h_{1}, \cdots, h_{k_{m}}\right\} \text { with }\|h\|=1 .
$$

This completes the proof of Theorem 1.2. 


\section{ACKNOWLEDGEMEnTs}

This research was supported by the Slovenian Research Agency grants P1-0292, N1-0064, J1-8131, J1-7025, and J1-6721, the Romanian National Authority for Scientific Research and Innovation grant CNCS-UEFISCDI, PN-III-P4-ID-PCE-20160130, the Science and Technology Research of the Henan Education Department grant 14A110011, and the National Natural Science Foundation of China grants 11326161 and 10971087.

\section{REFERENCES}

[1] E. Acerbi, G. Mingione, Regularity results for a class of functionals with nonstandard growth, Arch. Ration. Mech. Anal. 156 (2001), 121-140.

[2] E. Acerbi, G. Mingione, Regularity results for stationary electro-rheological fluids, Arch. Ration. Mech. Anal. 164 (2002), 213-259.

[3] C.O. Alves, S.B. Liu, On superlinear $p(x)$-Laplacian equations in $\mathbb{R}^{N}$, Nonlinear Analysis $\mathbf{7 3}$ (2010), 2566-2579.

[4] S. Antontsev, S. Shmarev, Elliptic equations and systems with nonstandard growth conditions: existence, uniqueness and localization properties of solutions, Nonlinear Analysis 65 (2006), 728-761.

[5] A. Ayoujil, On the superlinear Steklov problem involving the $p(x)$-Laplacian, Electron. J. Qual. Theory Differ. Equ. (2014), No. 38, 1-13.

[6] J.F. Bonder, N. Saintier, A. Silva, On the Sobolev embedding theorem for variable exponent spaces in the critical range, Journal of Differential Equations, 253 (2012), 1604-1620.

[7] J.F. Bonder, N. Saintier, A. Silva, Existence of solution to a critical equation with variable exponent, Ann. Acad. Sci. Fenn. Math., 37 (2012), 579-594.

[8] J. Chabrowski, Y.Q. Fu, Existence of solutions for $p(x)$-Laplacian problems on a bounded domain, J. Math. Anal. Appl. 306 (2005), 604-618.

[9] K.C. Chang, Critical Point Theory and Applications, Shanghai Scientific and Technology Press, Shanghai, 1986.

[10] Y. Chen, S. Levine, M. Rao, Variable exponent, linear growth functionals in image restoration, SIAM J. Appl. Math. 66 (2006), 1383-1406.

[11] A. Coscia, G. Mingione, Hölder continuity of the gradient of $p(x)$-harmonic mappings, C.R. Acad. Sci. Paris, Sér. I 328 (1999), 363-368.

[12] L. Diening, P. Harjulehto, P. Hästö, M. Růžička, Lebesgue and Sobolev Spaces with Variable Exponents, Lecture Notes in Mathematics, vol. 2017, SpringerVerlag, Berlin, 2011.

[13] X.L. Fan, Global $C^{1, \alpha}$ regularity for variable exponent elliptic equations in divergence form, J. Differential Equations 235 (2007), 397-417.

[14] X.L. Fan, On the sub-supersolution method for $p(x)$-Laplacian equations, $J$. Math. Anal. Appl. 330 (2007), 665-682. 
[15] X.L. Fan, Q.H. Zhang, Existence of solutions for $p(x)$-Laplacian Dirichlet problem, Nonlinear Anal. 52 (2003), 1843-1852.

[16] X.L. Fan, Q.H. Zhang, D. Zhao, Eigenvalues of $p(x)$-Laplacian Dirichlet problem, J. Math. Anal. Appl. 302 (2005), 306-317.

[17] X.L. Fan, D. Zhao, On the spaces $L^{p(x)}(\Omega)$ and $W^{m, p(x)}(\Omega)$, J. Math. Anal. Appl. 263 (2001), 424-446.

[18] Y.Q. Fu, The principle of concentration-compactness in $L^{p(x)}$ spaces and its application, Nonlinear Analysis 71 (2009), 1876-1892.

[19] L. Gasiński, N. Papageorgiou, Anisotropic nonlinear Neumann problems, Calc. Var. Partial Differential Equations 42 (2011), 323-354.

[20] L. Gasiński, N. Papageorgiou, A pair of positive solutions for the Dirichlet $p(z)$-Laplacian with concave and convex nonlinearities, J. Global Optim. 56 (2013), 1347-1360.

[21] A. El Hamidi, Existence results to elliptic systems with nonstandard growth conditions, J. Math. Anal. Appl. 300 (2004), 30-42.

[22] P. Harjulehto, P. Hästö, Ú. V. Lê, M. Nuortio, Overview of differential equations with non-standard growth, Nonlinear Anal. 72 (2010), 4551-4574.

[23] P. Harjulehto, T. Kuusi, T. Lukkari, N. Marola, M. Parviainen, Harnack's inequality for quasi-minimizers with non-standard growth conditions, J. Math. Anal. Appl. 344 (2008), 504-520.

[24] T. Kopaliani, Interpolation theorems for variable exponent Lebesgue spaces, J. Functional Analysis 257 (2009), 3541-3551.

[25] O. Kováčik, J. Rákosník, On spaces $L^{p(x)}(\Omega)$ and $W^{k, p(x)}(\Omega)$, Czechoslovak Math. J. 41 (1991), 592-618.

[26] G.B. Li, C.Y. Yang, The existence of a nontrivial solution to a nonlinear elliptic boundary value problem of $p$-Laplacian type without the AmbrosettiRabinowitz condition, Nonlinear Analysis 72 (2010), 4602-4613.

[27] S.B. Liu, On superlinear problems without the Ambrosetti and Rabinowitz condition, Nonlinear Analysis 73 (2010), 788-795.

[28] T. Lukkari, Singular solutions of elliptic equations with nonstandard growth, Math. Nachr. 282 (12) (2009), 1770-1787.

[29] M. Mihailescu, V.D. Rădulescu, D.D. Repovš, On a non-homogeneous eigenvalue problem involving a potential: an Orlicz-Sobolev space setting, J. Math. Pures Appl. 93 (2010), 132-148.

[30] M. Mihailescu, D.D. Repovš, Multiple solutions for a nonlinear and nonhomogeneous problem in Orlicz-Sobolev spaces, Appl. Math. Comput. 217 (2011), 6624-6632.

[31] O.H. Miyagaki, M.A.S. Souto, Superlinear problems without Ambrosetti and Rabinowitz growth condition, J. Differential Equations 245 (2008), 3628-3638.

[32] G. Molica Bisci, D. Repovš, Multiple solutions for elliptic equations involving a general operator in divergence form, Ann. Acad. Sci. Fenn. Math. 39 (2014), $259-273$. 
[33] P. Pucci, Q.H. Zhang, Existence of entire solutions for a class of variable exponent elliptic equations, J. Differential Equations 257 (2014), 1529-1566.

[34] V. Rădulescu, Nonlinear elliptic equations with variable exponent: old and new, Nonlinear Anal. 121 (2015), 336-369.

[35] V. Rădulescu, D. Repovš, Partial Differential Equations with Variable Exponents: Variational Methods and Qualitative Analysis, CRC Press, Taylor \& Francis Group, Boca Raton FL, 2015.

[36] V. Rădulescu, I. Stăncut, Combined concave-convex effects in anisotropic elliptic equations with variable exponent, Nonlinear Differential Equations and Applications (NoDEA) 22 (2015), 391-410.

[37] M. Růžička, Electrorheological Fluids: Modeling and Mathematical Theory, Lecture Notes in Mathematics 1748, Springer-Verlag, Berlin, 2000.

[38] S.G. Samko, Denseness of $C_{0}^{\infty}\left(\mathbb{R}^{N}\right)$ in the generalized Sobolev spaces $W^{m, p(x)}\left(\mathbb{R}^{N}\right)$, Dokl. Ross. Akad. Nauk 369 (1999), No.4, 451-454.

[39] M. Willem, W. Zou, On a Schrödinger equation with periodic potential and spectrum point zero, Indiana Univ. Math. J. 52 (2003), 109-132.

[40] X.C. Xu, Y.K. An, Existence and multiplicity of solutions for elliptic systems with nonstandard growth condition in $\mathbb{R}^{N}$, Nonlinear Analysis 68 (2008), 956968.

[41] N. Yoshida, Picone identities for half-linear elliptic operators with $p(x)$ Laplacians and applications to Sturmian comparison theory, Nonlinear Analysis 74 (2011), 5631-5642.

[42] A.B. Zang, $p(x)$-Laplacian equations satisfying Cerami condition, J. Math. Anal. Appl. 337 (2008), 547-555.

[43] Q.H. Zhang, A strong maximum principle for differential equations with nonstandard $p(x)$-growth conditions, J. Math. Anal. Appl. 312 (2005), 24-32.

[44] Q.H. Zhang, Existence and asymptotic behavior of positive solutions for variable exponent elliptic systems, Nonlinear Analysis 70 (2009), 305-316.

[45] Q.H. Zhang, C.S. Zhao, Existence of strong solutions of a $p(x)$-Laplacian Dirichlet problem without the Ambrosetti-Rabinowitz condition, Computers and Mathematics with Applications 69 (2015), 1-12.

[46] X.X. Zhang, X.P. Liu, The local boundedness and Harnack inequality of $p(x)$ Laplace equation, J. Math. Anal. Appl. 332 (2007), 209-218.

[47] J.F. Zhao, Structure Theory of Banach Spaces, Wuhan University Press, Wuhan, 1991 (in Chinese).

[48] V.V. Zhikov, Averaging of functionals of the calculus of variations and elasticity theory, Math. USSR. Izv. 29 (1987), 33-36.

[49] V.V. Zhikov, Existence theorem for some pairs of coupled elliptic equations, Doklady Mathematics 77 (2008), 80-84.

[50] C.K. Zhong, X.L. Fan, W.Y. Chen, Introduction to Nonlinear Functional Analysis, Lanzhou University Press, Lanzhou, 1998. 
(G. Li) College of Mathematics and Information Science, Zhengzhou University of Light Industry, Zhengzhou, Henan 450002, China

E-mail address: leagongpaper@yeah.net

(V.D. Rădulescu) Institute of Mathematics, Physics and Mechanics, 1000 Ljubluana, Slovenia \& Faculty of Applied Mathematics, AGH University of Science and Technology, 30-059 Kraków, Poland

E-mail address: vicentiu.radulescu@imfm.si

(D.D. Repovš) Faculty of Education and Faculty of Mathematics and Physics, University of Ljubljana, \& Institute of Mathematics, Physics and Mechanics, 1000 Ljubljana, SLOVENIA

E-mail address: dusan.repovs@guest.arnes.si

(Q. Zhang) College of Mathematics and Information Science, Zhengzhou University of Light Industry, Zhengzhou, Henan 450002, China

E-mail address: zhangqihu@yahoo.com 